\title{
A percepção do cuidador frente aos pacientes neurológicos na Clínica Escola de Fisioterapia da Unochapecó
}

Perception of caregiver in the neurological patients in the Clinic School of Physiotherapy of Unochapecó
FisiSenectus. Unochapecó Ano 7, n. 2 - Jul/Dez. 2019 p. $4-11$

\section{Gustavo Camargo.gustavocolpo@unochapeco.edu.br}

Acadêmico de Fisioterapia da Universidade Comunitária da Região de Chapecó (Unochapecó).

Douglas Carlos Tuni. tuni.douglas@outlook.com

Acadêmico de Medicina da Universidade Comunitária da Região de Chapecó (Unochapecó).

Kauana Inês Petzen. kauanapetzen@unochapeco.edu.br

Acadêmica de Fisioterapia da Universidade Comunitária da Região de Chapecó (Unochapecó).

Michele Minozzo dos Anjos. micheleminozzo@unochapeco.edu.br

Fisioterapeuta. Mestre em Biociências e Reabilitação (IPA). Docente do Curso de Fisioterapia da Universidade Comunitária da Região do Chapecó (Unochapecó).

Aline Martinelli Piccinini. aaline-martinelli@hotmail.com

Fisioterapeuta. Mestre em Ciências do Movimento pela Universidade Federal do Rio Grande do Sul (UFRGS). Mestre em Docência Universitária (UTN). Professora do Curso de Fisioterapia da Universidade Comunitária da Região do Chapecó (Unochapecó).

\section{Resumo}

Introdução: cuidadores assumem o papel do cuidado do paciente fisicamente incapacitado no contexto hospitalar e domiciliar e representam o elo entre paciente, família e equipe de saúde. A tarefa de cuidador de pacientes neurológicos é desgastante, pois ele assume a responsabilidade de dar suporte e assistir às necessidades em saúde da pessoa. Objetivo: conhecer as percepções dos cuidadores frente às dificuldades encontradas no cuidado diário de pacientes neurológicos. Metodologia: estudo qualitativo descritivo relacionado às percepções de 30 cuidadores de pacientes neurológicos que frequentam a Clínica Escola de Fisioterapia da Unochapecó. 0 critério de inclusão foi a aceitação dos cuidadores em participar da pesquisa, respondendo a um questionário de identificação e realizando uma entrevista semiestruturada acerca da percepção do cuidador frente ao paciente neurológico e às dificuldades diárias. As entrevistas foram gravadas e transcritas, abrangendo questões sobre o que é ser cuidador, como é o dia a dia com o paciente e qual a percepção do cuidador frente ao paciente neurológico. 0 diário de campo foi importante para relacionar a fala gravada com a percepção do entrevistador. Resultados: a média de idade foi de 38,6 anos, com predominância do gênero feminino (93\%). Pode-se notar características inerentes ao cuidado e ao cuidador, relacionadas ao amor, carinho e responsabilidade, além das dificuldades encontradas diariamente, como barreiras físicas domiciliares e a falta de acessibilidade de alguns locais públicos. Conclusão: o presente estudo realçou 0 despreparo dos cuidadores, que implica desvantagens para ambos (quem cuida e quem é cuidado). Com isso, buscam-se formas de instrumentalizar o cuidador.

\section{Palavras-chave:}

Neurologia; Saúde holística; Qualidade de vida. 


\begin{abstract}
Introduction: caregivers are individuals who assume the role of physically incapacitated patient care in the hospital and home context, and represent the link between the patient, the family and the health team. The task of caregivers of neurological patients is exhausting, since they assume the responsibility of providing support and attending to the health needs of the person. Objective: to know the caregivers' perceptions regarding the difficulties encountered in the daily care of neurological patients. Methodology: Descriptive qualitative study related to the perceptions of 30 caregivers of neurological patients attending the Unochapecó School of Physical Therapy. The inclusion criterion was the caregivers' acceptance to participate in the research, answering an identification questionnaire and conducting a semi-structured interview about the caregiver's perception of the neurological patient and the daily difficulties. The interviews was recorded and overwrote, covering questions about what it is to be a caregiver, the daily routine with the patient, and the caregiver's perception of the neurological patient. The field diary was important for relating recorded speech to the interviewer's perception. Results: the average age was 38.6 years, with a predominance of females (93\%). It may been noted inherent characteristics of care and caregiver, related to love, affection and responsibility, in addition to the difficulties encountered daily, such as home physical barriers and the lack of accessibility of some public places. Conclusion: the present study emphasized the lack of preparation of caregivers that implies disadvantages for both caregivers and caregivers. With this, one looks for ways to instrumentalize the caregiver.
\end{abstract}

\title{
Keywords:
}

Neurology; Holistic health; Quality of life.

\section{Introdução}

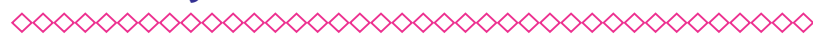

Quando se trata de cuidado, precisa-se buscar conceitos e fundamentos que o validem e o determinem dentro de um estudo, pesquisa e trabalhos científicos específicos. Martin Heidegger (18891976), em seu livro Ser e tempo (1927), vê, "do ponto de vista existencial, que o cuidado se acha $a$ priori, antes de toda atitude e situação do ser humano, o que sempre significa dizer que ele se acha em toda atitude e situação de fato"1.

Em se tratando da área da saúde, principalmente na relação clínica, "o cuidado deve ressaltar os elementos individuais da interação profissional-paciente, valorizando as questões que interferem no processo saúde-doença" ${ }^{2}$. Na mesma ótica, Pinheiro 3 afırma que o cuidado "é uma ação integral que tem significados e sentidos voltados para compreensão de saúde como direito de ser, tratando, respeitando, acolhendo e atendendo o ser humano em seu sofrimento".

Levando em conta a função de cuidador, define-se esse cargo como aquele realizado por indivíduos responsáveis pelo cuidado de pessoas doentes ou dependentes, auxiliando em atividades da vida diária, guiando aos serviços de saúde ${ }^{4}$. Desse modo, há um enorme percentual de incapacidade funcional entre os pacientes, pois as ações do dia a dia tornam-se impossibilitadas, e os riscos de hospitalização crescem ${ }^{5}$. Além disso, alterações clínicas cardiorrespiratórias e sintomas depressivos ${ }^{6}$ também são comuns entre essa população.

Além das dificuldades para execução de tarefas motoras, os pacientes neurológicos podem apresentar alterações cognitivas, o que leva a níveis alterados de excitação, vigília reduzida, deficiência de memória, agitação, desinibição, euforia, variação na motivação e déficits de linguagem, necessitando de terapia cognitivo-comportamental $(\mathrm{TCC})^{7}$.

Por esses motivos, o cuidado nesses casos é necessário e o papel dos cuidadores é de suma importância, sejam eles formais ou informais. Os cuidadores formais exercem sua função em horários preestabelecidos, atuando a domicílio. Já os informais podem ser membros da família, amigos, vizinhos, sem benefício monetário, de maneira integral $^{8}$.

O trabalho dos cuidadores é de suma importância para a manutenção e melhora nas condições de saúde do paciente. São eles que assumem todas 
as funções que envolvem o indivíduo cuidado, entretanto, na maioria dos casos, desempenham esse papel sem nenhum tipo de ajuda. O olhar das pessoas muitas vezes se fecha ao paciente, esquecendo-se da sobrecarga na qual o cuidador se encontra. 0 cuidador estressado corre o risco de transferir sua insatisfação para o paciente, além de poder desenvolver problemas de saúde, como lombalgia, dor em membros inferiores, crises de ansiedade e hipertensão arterial, por exemplo ${ }^{9}$.

Dessa forma, é preciso entender o olhar do cuidador e suas perspectivas, pois não há cuidador absoluto. 0 cuidador também precisa ser cuidado. Segundo Boff, o ato de cuidar está na raiz do ser humano. Cuidar é mais do que um ato, é uma atitude que gera muitos outros atos. Sendo assim, cuidar de quem cuida é valorizar a definição de cuidado na sua totalidade, tendo como resultado final a melhora exponencial na saúde de todos os envolvidos.

Diante desse contexto, surge como necessidade de pesquisa e investigação o entendimento acerca da percepção do cuidador frente às dificuldades encontradas no cuidado diário dos seus pacientes neurológicos. 0 objetivo deste estudo foi conhecer as percepções dos cuidadores frente às dificuldades encontradas no cuidado diário de pacientes neurológicos.

\section{Métodos}

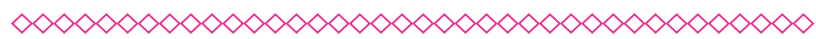

Este estudo é de caráter quantitativo descritivo e envolveu informações relacionadas às percepções dos cuidadores de pacientes neurológicos que frequentam a Clínica Escola de Fisioterapia Professora Sabrina Fiorentin Sfreddo, da Universidade Comunitária da Região de Chapecó (Unochapecó).

Os cuidadores que concordaram em participar responderam a um questionário de identificação e, em seguida, realizaram uma entrevista semiestruturada acerca da percepção do cuidador frente ao paciente neurológico e às dificuldades diárias. A coleta e análise dos dados foi realizada através da triangulação das informações colhidas em entrevistas semiestruturadas, diário de campo e literatura.
A entrevista semiestruturada tem por objetivo coletar informações sobre determinado tema científico, sendo uma das estratégias mais usadas no trabalho de campo. As entrevistas foram gravadas e posteriormente transcritas. 0 material foi lido, explorado e agregado em categorias: percepções dos cuidadores, relacionadas ao reconhecimento do papel desempenhado; responsabilidades no cuidado diário; e identificação do conhecimento dos cuidadores sobre as doenças neurológicas. Ao final do estudo, foi entregue um material informativo como forma de auxílio aos cuidadores.

O diário de campo foi utilizado como um instrumento de pesquisa, no qual o pesquisador registrou diariamente suas impressões pessoais e comportamentos contraditórios com as falas e descreveu tudo aquilo que observou e que foi relevante ao tema da pesquisa ${ }^{10}$.

Este estudo está de acordo com as orientações éticas de pesquisa que resguardam o respeito às pessoas, conforme estabelece a Resolução $n^{0}$ 466/12, do Conselho Nacional de Saúde. Foi aprovado pelo Comitê de Ética em Pesquisa (CEP) da Unochapecó, que gerou o número do parecer 2.443.414, o qual viabilizou a concretização desta pesquisa.

\section{Resultados e discussão}

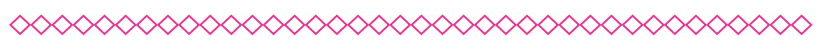

A realidade deste estudo mostra que os dados qualitativos e quantitativos estão relacionados uns aos outros. 0 universo de estudo foi constituído por cuidadores de pacientes neurológicos que são, ou foram, atendidos na Clínica Escola de Fisioterapia Professora Sabrina Fiorentin Sfreddo, da Unochapecó. Participaram do estudo 30 pessoas, tendo uma média de idade de 38,6 anos, com um desvio padrão de 10,82. Verificou-se que, do total de cuidadores, a maioria, 28, é do gênero feminino (93\%).

Além disso, 13 (43,3\%) são mães dos pacientes, seguidas de $11(36,6 \%)$ outros cuidadores (amigos, vizinhos); três (10\%) são filhos(as); apenas dois $(6,6 \%)$ são pais que exercem a função de cuidar; e, por fim, um (3,3\%) é técnico em enfermagem. 
Esses dados reforçam a noção de que as muIheres são os principais cuidadores, desde o início, com a construção familiar, até os momentos difíceis de hospitalização e cuidados domésticos ${ }^{11}$. Além disso, nota-se uma prevalência de cuidadores informais, assim como apontado por outros estudos da área ${ }^{8,11,12}$.

Dos cuidadores entrevistados, 17 (76,6\%) despendem 120 horas ou mais no cuidado semanal de seus pacientes. Vinte $(66,6 \%)$ deles exerciam essa profissão há um ano ou mais, e somente seis (20\%) já cuidaram de outros pacientes. Porém, apenas dois $(6,6 \%)$ cuidam de outros pacientes concomitantemente, ou seja, tutelam dois ou mais pacientes semanalmente.

A presente pesquisa corrobora vários estu$\operatorname{dos}^{13,14}$, quando se evidencia a carga horária de trabalho do cuidador. 0 que se percebe é uma sobrecarga diária, muitas vezes ocupando-se 24 horas por dia na realização dos afazeres.

Além disso, outro ponto importante a destacar é a falta de experiência no cuidado, pois, como evidenciado nesta pesquisa, somente seis (20\%) entrevistados já cuidaram de outro paciente. Isso é preocupante, uma vez que, conforme Karsch ${ }^{15}$, "o cuidador familiar [...] precisa de orientações de como proceder nas situações mais difíceis". Nesse caso, entende-se que é fundamental a presença de profissionais da área da saúde para capacitar os responsáveis pelo paciente, ou seja, os cuidadores.

No exercício do cuidar, muitos acabam abandonando seu trabalho. Neste estudo, houve uma equiparação, tendo uma leve predominância dos que não trabalham ou deixaram de trabalhar (53\%) para cuidar de um paciente. Um estudo de Brigola et al. ${ }^{16}$ mostrou que $76 \%$ dos cuidadores não exerciam atividades remuneradas, ou seja, estavam focados unicamente no paciente, nesse caso, um familiar.

Dos 30 cuidadores envolvidos neste estudo, oito $(26,6 \%)$ fazem uso de medicação, sendo que mais da metade são medicamentos ansiolíticos e antidepressivos. Quanto ao sono, 56,6\% informaram dormir por mais de oito horas diárias. 0 estudo de Brigola et al. ${ }^{16}$ também trata da medicação dos cuidadores, sendo comum o uso de, no mínimo, um remédio. Ademais, relata-se insônia, o que não pode ser comparado ao presente estudo.
Quanto ao número de cuidadores por paciente, identificou-se que 16 (53\%) pacientes necessitam de mais de um cuidador para desempenhar as atividades diárias. Essa mesma porcentagem está relacionada à idade dos pacientes, com pequena prevalência na faixa de 0 a 25 anos.

A pesquisa de abordagem qualitativa aceita outros meios de coleta de dados. Foi por meio de um roteiro de perguntas realizadas - entrevista semiestruturada - que se procurou conhecer as percepções dos cuidadores frente às dificuldades encontradas no cuidado diário de pacientes neurológicos. As entrevistas semiestruturadas foram analisadas a partir do agrupamento de temáticas que emergiram das falas dos cuidadores.

A partir dessas entrevistas, buscou-se destacar os principais achados do estudo, tentando estabelecer redes de aproximação entre os eixos deste trabalho assim agrupados: o que é cuidado para você, o que é ser cuidador para você? Como você se sente cuidando de um paciente com problema neurológico? Como é seu dia a dia cuidando de seu paciente neurológico? Quais as principais dificuldades que você encontra no cuidado diário? Há ajuda por parte de outras pessoas no cuidado diário do paciente? Qual a sua percepção sobre o problema neurológico do seu paciente?

Utilizou-se o diário de campo para registros escritos e observações de visitas e contatos (pessoais ou telefônicos) realizados durante o estudo. Assim, nesse material, de forma minuciosa, registraram-se as percepções, impressões e conversas consideradas importantes no encontro com os cuidadores na Clínica Escola de Fisioterapia Professora Sabrina Fiorentin Sfreddo, da Unochapecó.

Com o intuito de não identificar os usuários e preservá-los, optou-se por utilizar o nome de frutas para caracterizá-los. Então, os nomes dados foram: Abacate, Abacaxi, Açaí, Ameixa, Amora, Cereja, Kiwi, Laranja, Maçã, Mamão, Manga, Maracujá, Melão, Morango e Pêra.

Tomando por base as perguntas referidas anteriormente e utilizadas na construção da pesquisa, procura-se sintetizar as respostas e anexar as falas dos cuidadores.

Iniciando pelo primeiro critério - o cuidado -, de acordo com os entrevistados, este está relacionado 
a um ato de amor, carinho, ajuda, zelo, enfim, um completo e complexo apoio físico e emocional para com o seu paciente. A seguir, apresentam-se os discursos de alguns deles: "É ajudar ao máximo..., tendo amor e carinho." (Amora). "Cuidá é auxiliá, tem que tê os remédios nas horas certinhas... porque quem cuida ela ama... zela." (Ameixa). "Cuidado é tudo, né... desde banho, vesti, alimentá, coloca pra dormi." (Maçã).

Sob a mesma óptica, mas de um ponto de vista mais preparado ao cuidado, há a presença dos abrigos de crianças. Destaca-se a afırmação de uma cuidadora, segundo a qual o trabalho realizado por eles visa substituir a família, com preocupações nas Atividades de Vida Diárias (AVDs), tais como alimentação, vestuário e estudos. A entrevistada ressaltou ainda que "é o cuidado como o de mãe e de pai".

Em se tratando da área da saúde, "cuidado é uma ação integral que tem significados e sentidos voltados para compreensão de saúde como direito de ser, tratando, respeitando, acolhendo e atendendo o ser humano em seu sofrimento" ${ }^{3}$.

Na sequência da entrevista, ao serem perguntados sobre "o que é ser cuidador para você?", os entrevistados mencionaram a importância da disposição integral do tempo, estimulando o paciente e auxiliando-o na dificuldade pela qual está passando, através do zelo, do afeto, do conforto, mas, acima de tudo, com responsabilidade: "O cuidador é a pessoa que tá ali à disposição, o tempo intero, né... É auxiliá, incentiva... além de ajuda você precisa estimulá." (Amora). "É atende, dá todo o necessário, carinho, afeto, atenção, os cuidados básicos necessários." (Abacate). "Fica diariamente cuidando da pessoa... zela pelo bem-estar, cuidá dela, né." (Ameixa). "É atende, ajuda... nas limitações dela... dá o máximo de conforto, qualidade de vida." (Mamão). "É ter responsabilidade." (Abaca$x i)$.

Segundo o Descritor em Ciências da Saúde $(\operatorname{DeCS})^{17}$, cuidadores são pessoas que proveem cuidado para aqueles que precisam de supervisão ou assistência, seja em casa, em hospital ou em instituição, não se restringindo a profissionais da área da saúde, mas considerando também outras pessoas, tais como pais, mães, cônjuges e demais familiares.
Alguns estudos ${ }^{11,12,16,18}$ têm mostrado que 0 perfil dos cuidadores é constituído de mulheres, donas de casa, que possuem relação de parentesco e que convivem diretamente com a pessoa cuidada, sendo esses fatores os mais determinantes para a assistência domiciliar. Isso vai ao encontro deste estudo, pois, do total de cuidadores entrevistados, $95,8 \%$ eram mulheres, as quais afirmaram que, em algum momento, precisaram abster-se do serviço formal remunerado para cuidar do paciente e dos afazeres da casa.

Referente ao sentimento em cuidar de um paciente neurológico, os entrevistados reagiram de diversas formas, alguns frisando a dificuldade no cuidado, outros tristes pela situação em que se encontra o paciente. Há também aqueles que, mesmo com as complicações clínicas, sentem-se esperançosos pelo grau de evolução que visualizam no paciente ao qual prestam o cuidado: "É difícil e gratificante ao mesmo tempo, porque a gente percebe grandes avanços." (Cereja). "Me sinto deprimido... por vê o que algumas crianças fazem, e tu não pode corre, não pode brincá." (Açaí).

Pedroso e Motta ${ }^{19}$, em seus estudos, enfatizam a "necessidade de um conhecimento do contexto de vida de um indivíduo para que se entenda que a concepção de vulnerabilidade modifica de pessoa para pessoa, determinada por diversos fatores, sejam eles de que ordens forem".

Quando perguntado aos cuidadores "como é seu dia a dia cuidando de seu paciente neurológico?", os entrevistados realçaram a agitação, seguida de uma série de ações que necessitam ser concluídas. Em contrapartida, alguns se referiram às oscilações do paciente, que não apresenta uma evolução constante, além das limitações que cada um possui, já que são várias, como se percebe nas falas a seguir: "A gente fica meio frustrado, porque... elas não têm evolução que a gente gostaria... às vezes elas evoluem no quadro... às vezes adoece, volta tudo." (Abacate). "Não consegue vestir uma roupa... tudo com ajuda... na fala a gente não entende muito... escrever ela não lembra das letras... tu pede calma, ela não tem... é bem difícil." (Kiwi).

0 ato de cuidar, embora os entrevistados não tenham citado, gera um cansaço físico e emocional aos cuidadores, ocasionando estresse crônico, que pode afetar em maior ou menor grau a saúde do 
cuidador 20,21,22. Além disso, as frustrações diárias dos cuidadores, em decorrência da estagnação na reabilitação ou da dificuldade em ações básicas, geram quadros álgicos, constipação e, a longo prazo, hipertensão arterial sistêmica ${ }^{16}$.

Foi solicitado aos cuidadores que identificassem as dificuldades encontradas no cuidado diário de um paciente neurológico. 0 principal ponto levantado pelos entrevistados foi a falta de acessibilidade das cidades, relacionada à infraestrutura, concomitante ao desrespeito da população, excluindo ao invés de incluir as pessoas. Além disso, há o despreparo dos próprios cuidadores com as barreiras físicas do ambiente em que se encontra 0 paciente: "Você percebe a dificuldade que as pessoas, com deficiências ou com limitações, possuem... só quem passa por essa situação... sabe a dificuldade que se tem... as cidades são preparadas pras pessoas normais... não se tem estrutura pra atende..." (Amora). "Não tê um lugar pra estaciona... ou chega no carro e já tê alguém estacionado, no lugar do cadeirante, que às vezes nem é cadeirante." (Açaí). "A casa... não tem adaptações pra ela... é mais dificuldade pra leva no banhero..." (Laranja).

Em contrapartida ao exposto pelos entrevistados, observou-se que a falta de informação e o despreparo das redes de suporte aos cuidadores são as principais contrariedades identificadas ${ }^{10,12}$. Este estudo reafirma que há sim um despreparo dos cuidadores no enfrentamento da situação, o que pode se dever à pouca intervenção das secretarias de saúde em ofertar cursos profissionalizantes e formadores de cuidadores capacitados para o cuidado.

Quando perguntados se "há ajuda por parte de outras pessoas no cuidado diário de seu paciente?", os entrevistados, com exceção da cuidadora do abrigo, apontaram que os familiares e amigos auxiliam, ou simplesmente não recebem assistência. Já a cuidadora que tem seu familiar no abrigo relata que tem uma equipe multiprofissional de prontidão para realizar os atendimentos e amparar os que se fazem presentes: "Tem toda uma equipe... tem monitores, auxiliares de enfermagem... psicólogo... assistente social." (Abacate). "Sempre teve alguém comigo... além dele tem todo o resto... filhos, casa, o dia a dia... teve a inversão de papéis... amigos e família... faz toda a diferença... te dá um suporte... porque teu emocional está abalado, né." (Amora). "Não tem, é só eu." (Manga).

De certa forma, a presença ou ausência de assistência externa ao cuidado reflete o parâmetro mais comum relacionado ao cuidado, que é a utilização de cuidadores informais, em âmbito domiciliar, que desempenham cuidado não profissional e sem receber nenhuma remuneração, podendo ser pessoas da família, amigos e vizinhos $^{8}$. Muitos estudos ${ }^{12,16}$ apontam a presença de ajuda afetiva, que, na sua grande maioria, serve como fonte de força e energia para manter o papel de ser cuidador.

A questão 7 indagou os participantes sobre "qual a sua percepção sobre o problema neurológico do seu paciente". Alguns entrevistados apontaram certo desconhecimento a respeito do diagnóstico clínico do seu paciente, outros afirmaram perceber evolução lenta e gradual, e há aqueles que planejam um futuro positivo e de conquistas a seus pacientes: "Não tem... bem certo o que ela tem... mas pode havê melhora, pode não havê." (Maracujá). "Pelo que ele passo, ele tá bem hoje... o grau de dicefalia deu leve... ele fico com as sequela..." (Melão). "Ela tá evoluindo bem... com oito dias ela mexeu ombrinho, antes dos três ela começou mexe até no cotovelo... agora ela ergue o braço, falta movimento na mão." (Maçã). "No começo fiquei bem apavorada... a gente... nunca viu fala dessa doença... agora essa questão tá melhorando... eu tenho uma perspectiva já bem alta com o meu filho." (Morango).

A percepção sobre o problema neurológico de um paciente está relacionada à compreensão do cuidador acerca do atendimento de suas necessidades e à expectativa sobre os cuidados de saúde ${ }^{23,24}$. Portanto, não há um consenso acerca da percepção do cuidador frente ao quadro neurológico de seu paciente, pois cada afecção merece uma conduta específica, já que a neurologia é bastante ampla.

Por fim, quando perguntados sobre algum comentário adicional na entrevista, os cuidadores levantaram um questionamento quanto à importância da ajuda das pessoas, o aspecto monetário na evolução mais rápida de uma pessoa, além da coragem e força para encarar essas situações: "Eu jamais posso dizê qui eu não vô passa por isso... a 
gente não sabe... e nesse momento você vê o quanto é importante você conta com a ajuda das pessoas." (Amora). "A questão financeira na qualidade de vida do paciente... ajuda, muito." (Mamão). "Eu nunca pensei que eu fosse... cuida de alguém assim... a gente dá mais valor pras pessoas que cuidam de alguém quando a gente passa por isso..." (Pêra).

A saúde é um direito de todos e um dever do Estado, como reforça a Constituição de 1988, em seu artigo 196: "garantido mediante políticas sociais e econômicas que visem à redução do risco de doença e de outros agravos e ao acesso universal e igualitário às ações e serviços para sua promoção, proteção e recuperação"25.

\section{Conclusão}

$\infty \times \infty \times \infty \times \infty \times \infty \times \infty \times \infty \times \infty \times \infty \times \infty \times \infty \times \infty \times \infty$

Tendo em vista a aplicação metodológica e os resultados obtidos através das entrevistas, infere-se que os cuidadores, sendo predominantemente informais, são despreparados para cuidar de pacientes, já que, na maior parte dos casos, são familiares ou pessoas próximas.

O conhecimento acerca das afeç̧ões desses pacientes também é precário, merecendo mais atenção, pois, quando se trata de doenças do Sistema Nervoso Central, o risco de sequelas e restrições no movimento é elevado. Além disso, vale ressaltar o papel dos profissionais na melhora do quadro clínico, pois quanto antes diagnosticada uma patologia e realizado o encaminhamento para reabilitação, melhor será o prognóstico do paciente e menor o sofrimento familiar.

Assim sendo, a qualidade de vida dos cuidadores sofrerá um impacto mais suave, pois terão a possibilidade de reformular o cronograma diário, adequando melhor as atividades a serem realizadas, tanto em favor do paciente quanto em prol de si mesmos.

Sugere-se a realização de estudos complementares, que considerem um maior número de cuidadores e que envolvam outras áreas de atendimento, para que se possa correlacionar os achados deste estudo com resultados futuros.

\section{Referências}

$\alpha<\infty<\infty<\infty<\infty<\infty<\infty<\infty<\infty<\infty<\infty<\infty<\infty<\infty<\infty<\infty<$

1. Boff L. Saber cuidar, ética do humano compaixão pela terra. 9. ed. Rio de Janeiro: Vozes; 2003.

2. Carnut L. Cuidado, integralidade e atenção primária: articulação essencial para refletir sobre o setor saúde no Brasil. Saúde Debate. 2017 out./ dez;41(115):1177-86.

3. Pinheiro R. Cuidado em saúde. [acesso em 2018 abr. 14]. Disponível em: http://www.epsjv. fiocruz. br/dicionario/verbetes/cuisau.html.

4. Diniz MAA, Melo BRS, Neri KH, Casemiro FG, Figueiredo LC, Gaioli CCLO et al. Estudo comparativo entre cuidadores formais e informais de idosos. Ciênc \& Saúde Col. 2018;23(11):3789-98.

5. Brito KQD, Menezes TN, Olinda RA. Functional disability: health conditions and physical activity practice in older adults. Rev Bras Enferm. 2016;69(5):773-80.

6. Virtuoso JJS, Martins CA, Roza LB, Paulo TRS, Ribeiro MCL, Tribess S. Prevalência de incapacidade funcional e fatores associados em idosos. Texto Contexto Enferm. 2015 abr./ jun;24(2): 521-9.

7. Oliveira C, Argimon I, Irigaray T, Moraes A, Piccoloto N. Terapia cognitivo-comportamental em pacientes neurológicos: uma revisão sistemática. Rev Bras Ter Comp e Cogn. 2015.

8. Martins G, Corrêa L, Caparrol AJS, Santos PTA, Brugnera LM, Gratão ACM. Características sociodemográficas e de saúde de cuidadores formais e informais de idosos com Doença de Alzheimer. Esc Anna Nery 2019;23(2).

9. Schnaider TB, Silva JV, Pereira MAR. Cuidador Familiar de Paciente com afeç̧ão neurológica. Rev Saúde Soc. 2009, 18(2): 284-292.

10. Lima TCS, Mioto RGT, Dal Prá KR. A documentação no cotidiano da intervenção dos assistentes sociais: algumas considerações acerca 
do diário de campo. Revista Textos \& Contextos. Porto Alegre, 2007; 6 (1): 93-104.

11. Jesus ITM, Orlandi AAS, Zazzetta MS. Sobrecarga, perfil e cuidado: cuidadores de idosos em vulnerabilidade social. Rev Bras Geriatr Gerontol. 2018 Mar-Abr, 21(2).

12. Araujo JS, Vidal GM, Brito FN, Gonçalves DCA, Leite DKM, Dutra CDT, Pires CAA. Perfil dos cuidadores e as dificuldades enfrentadas no cuidado ao idoso, em Ananindeua, PA. Rev. Bras. Geriatr. Gerontol. Rio de Janeiro, 2013; 16(1):149158

13. Tsai PC, Yip PK, Tai JJ, Lou ME. Needs of family caregivers of stroke patients: a longitudinal study of caregivers' perspectives. Patient Prefer Adherence. 2015;9:449-57.

14. Fernandes CS, Angelo M. Cuidadores familiares: o que eles necessitam? Uma revisão integrativa. Rev Esc Enferm. 2016;50(4):675682.

15. Karsch UM. Idosos dependentes: famílias e cuidadores. Cad. Saúde Pública. 2003 MaioJun,19(3):861 - 866.

16. Brigola AG, Luchesi BM, Rossetti ES, Mioshi E, Inouye $K$, Pavarini SCI. Perfil de saúde de cuidadores familiares de idosos e sua relação com variáveis do cuidado: um estudo no contexto rural. Rev. Bras. Geriatr. Gerontol. Rio de Janeiro, 2017; 20(3): 410-422.

17. BVS, DeCS - Descritores em Ciências da Saúde. Acessado em 16 de jul. de 2019. Disponível em http://decs.bvs.br/cgi-bin/ wxis1660.exe/decsserver/.

18. Yavo IS, Campos EMP. Cuidador e cuidado: o sujeito e suas relações no contexto da assistência domiciliar. Psicol Teor Prat. 2016, 18(1).

19. Pedroso MLR, Motta MGC. A compreensão das vulnerabilidades socioeconômicas no cenário da assistência de enfermagem pediátrica. Rev Gaúcha Enferm. 2010, 31(2):218-24.

20. Caldeira RB, Neri AL, Batistoni SST, Cachioni M. Variáveis associadas à satisfação com a vida em cuidadores idosos de parentes também idosos cronicamente doentes e dependentes. Rev Bras Geriatr Gerontol. 2017; 20(4):502-15.

21. Fuhrmann AC, Bierhals CCBK, dos Santos NO, Paskulin LMG. Association between the functional capacity of dependant elderly people and the burden of family caregivers. Rev Gaúcha Enferm. 2015;36(1):14-20.

22. Loureiro LSN, Fernandes MGM, da Nóbrega MML, Rodrigues RAP. Sobrecarga em cuidadores familiares de idosos: associação com características do idoso e demanda de cuidado. Rev Bras Enferm. 2014;67(2):227-32.

23. Ferreira DPC, Coriolano MGWS, Lins CCSA. Knowledge as a tool to promote care of the elderly with Parkinson's disease. J Nurs UFPE. 2016, 10(12):4628-36.

24. Ferreira DPC, Coriolano MGWS, Lins CCSA. The perspective of caregivers of people with Parkinson's: an integrative review. Rev. Bras. Geriatr. Gerontol. 2017, 20(1):103-114.

25. BRASIL. Constituição da República Federativa do Brasil, de 05 de outubro de 1988. Acesso em: 16 de jul. de 2019. Disponível em: https://www.senado.leg.br/atividade/const/ con1988/CON1988_05.10.1988/CON1988.asp. 